\title{
INVESTIGACIONES
}

\section{Formación del profesorado universitario en metodologías y su incidencia en el aula}

\author{
Training of university teaching staff \\ in methodologies and their impact in the classroom
}

\author{
David Jiménez Hernández ${ }^{a}$, Juan José González Ortiz , \\ María Tornel Abellán ${ }^{c}$ \\ ${ }^{a}$ Universidad Católica San Antonio de Murcia \\ Correo electrónico: djimenez361@ucam.edu \\ ${ }^{\mathrm{b}}$ Universidad Católica San Antonio de Murcia \\ Correo electrónico: jjgonzort@ucam.edu \\ ${ }^{c}$ Universidad Católica San Antonio de Murcia \\ Correo electrónico: mtornel@ucam.edu
}

\section{RESUMEN}

La formación del profesorado universitario ha sido una preocupación constante en los procesos de actualización docente. Este artículo, basado en los resultados de una encuesta de elaboración propia, pretende establecer una relación entre el tipo de formación recibida por 501 docentes universitarios de la Región de Murcia con el empleo posterior de métodos de enseñanza en el aula. A través de los resultados se pudo comprobar que una mayor formación didáctica de los docentes en métodos de enseñanza se relaciona con un mayor empleo de éstos. Además, la formación inicial influye en el mayor empleo de estos métodos a nivel general; específicamente los ingenieros superiores utilizan más la resolución de ejercicios y problemas que el resto de profesionales de otras áreas de conocimiento.

Palabras clave: métodos de enseñanza, enseñanza y formación, educación superior, innovación pedagógica

\begin{abstract}
The training of university teachers has been a constant concern in the processes of teacher development. A variety of training programs for university teachers have been developed within the European Higher Education Area (EHEA). This paper, based on the results obtained from a self-developed survey, aims at establishing a connection between different type of training received to 501 university teachers in the Region of Murcia and the subsequent use of teaching method in the classroom. Results suggested that didactic training on teaching methods is linked to their greater use. Furthermore, initial training also influences on a greater use of these methods in normal contexts; particularly, problem-solving activities are more frequently used by engineers than by professionals in other fields.
\end{abstract}

Key words: teaching methods, teaching and training, higher education, teaching method innovations 


\section{INTRODUCCIÓN}

La formación pedagógica del profesorado ha sido una de las cuestiones centrales en la renovación universitaria y así lo acreditan diferentes actividades llevadas a cabo en el despliegue del nuevo contexto universitario. Insertados en acciones estratégicas de las Facultades y Titulaciones, permiten, además de satisfacer las necesidades e intereses del profesorado, facilitar la adquisición de conocimientos, capacidades y competencias para el ejercicio de la docencia en contextos y tiempos complejos. Se fundan en el supuesto de que, para promover la calidad educativa conveniente, es preciso desarrollar un determinado modelo de profesorado y, en términos más amplios, de universidad (Ladwig, 2005). En palabras de Escudero y Portela (2015), las políticas de formación suponen determinados compromisos teóricos y prácticos con la construcción de la profesionalidad docente y su ejercicio. De ahí la pertinencia de una investigación que contribuya a conocer qué formación se está promoviendo y cuáles pueden ser sus efectos o incidencia en la mejora y calidad de la enseñanza (Kivunja, 2014).

Las actividades y metodologías formativas, entendidas como oportunidades para el aprendizaje docente, son elementos nucleares en el desarrollo profesional y, quizás, también de la enseñanza y aprendizaje que ocurre en las aulas. Por lo tanto, los propósitos de este estudio se dirigen a responder a diversas cuestiones sobre la formación del profesorado y su influencia en la docencia, con especial atención al empleo de diferentes métodos de enseñanza, puesto que la formación en metodología docente requiere asumir modalidades que, teniendo en cuenta el sentido comunitario y el contenido de formación del profesorado, se orienten a contextos concretos de práctica docente (López, 2016).

\section{FORMACIÓN DEL PROFESORADO UNIVERSITARIO EN METODOLOGÍAS DE ENSEÑANZA}

El interés por cuestiones didácticas y la propia variabilidad metodológica en la universidad se han visto favorecidas por la llegada del Espacio Europeo de Educación Superior (EEES), el sistema europeo de créditos, el cambio hacia un enfoque educativo centrado prioritariamente en el aprendizaje autónomo del alumnado, en el desarrollo de competencias y el aprendizaje a lo largo de la vida.

La dificultad estriba en que esta propuesta, además de traer preguntas e inseguridades, ha de llevarse a cabo en un contexto tradicionalmente escéptico con los cambios. Por esta razón, está siendo acompañada por una actualización y formación docente al servicio de las necesidades sociales y de la propia organización que asume la tarea de cambio y mejora. Por eso, Monereo (2013) justifica la formación docente por la necesidad que tienen las universidades de responder a desafíos actuales.

La formación del profesorado universitario es considerada un factor decisivo para el éxito de las transformaciones educativas, al prevenir carencias asociadas e impulsar la implicación del profesorado en el proceso (Rabadán \& Hernández, 2012). En el documento "Propuestas para la Renovación de las Metodologías Educativas en la Universidad" del Ministerio de Educación y Ciencia (2006), se vincula también el cambio metodológico a la formación de los docentes. Gallardo (2009-2010) y González, Macías, Rodríguez \& Aguilera (2009) discurren que el cambio hacia un paradigma educativo basado en métodos 
y aprendizajes activos necesita una formación inicial y permanente del profesorado universitario.

Por otro lado, aunque la formación es un elemento vital para el cambio, por sí misma no garantiza la mejora de la docencia, son los procesos que comprometen a varias instancias institucionales y afectan al diseño del proyecto formativo de las instituciones los que elevan el impacto de la innovación (Zabalza, Cid \& Trillo, 2014).

La formación permite al profesorado estar informado de las últimas tendencias y perspectivas teóricas existentes sobre la enseñanza y el aprendizaje de su disciplina, al mismo tiempo que adquiere las competencias que le permitirán reunir, analizar y comunicar evidencias significativas de su práctica docente y de su efectividad como profesor (Jarauta y Medina, 2012).

\section{METODOLOGÍAS DOCENTES Y RENOVACIÓN PEDAGÓGICA}

Tal y como se ha expuesto, el nuevo panorama universitario ha supuesto un cambio en todas sus dimensiones y la revisión de aspectos cruciales como los contenidos de las materias, la diversidad metodológica, recursos didácticos, estrategias de evaluación, organización de los sistemas de tutoría y asesoramiento de la autonomía del estudiante (González \& Raposo, 2008). La magnitud y profundidad de este cambio ha puesto de manifiesto la necesidad de replantear la formación docente del profesorado universitario (Madrid, 2005).

Existen algunas exigencias teóricas asociadas al proceso de convergencia europea que se han constituido como referentes para la formación y el perfeccionamiento docente. En lo que respecta al aprendizaje activo de los estudiantes, el profesorado necesita conocer y manejar estrategias metodológicas que potencien y favorezcan su participación, su trabajo autónomo y el desarrollo competencial e integral de los estudiantes (De la Calle, 2004).

Michavila (2005) propuso un análisis que se centre en las debilidades que hay que combatir. Su propuesta va dirigida a la dicotomía entre los individualismos en la adopción de estrategias metodológicas y su escaso reconocimiento en los méritos docentes. Verdaderamente es un hándicap que los docentes que más trabajen para buscar la renovación de los contenidos y metodologías empleadas, obtengan poco reconocimiento y su dedicación no sea valorada en la promoción profesional.

Como se viene aludiendo, existe una vinculación lógica entre los métodos de enseñanza, la necesaria formación del profesorado para su aplicación y la renovación pedagógica que lleva asociada su uso (Gore, Griffiths \& Ladwig, 2004). Tanto es así, que la mejora de la calidad del sistema universitario pasa por la reflexión de tres aspectos (Arribas, Carabias \& Monreal, 2010): el desarrollo de competencias docentes y profesionales, la utilización de metodologías activas como medio de renovación de la práctica docente universitaria tradicional y la implementación de un sistema de evaluación formativo. Pozo, Bretones, Martos \& Alonso (2011) reiteran en que la metodología empleada tiene un valor significativo en el nuevo contexto universitario, confirmando que los estudiantes cuyos profesores trabajan en metodologías centradas en su aprendizaje desarrollan estrategias de aprendizaje de más calidad y con enfoques más profundos que con aquellos que recurrían a métodos tradicionales dirigidos a la enseñanza (Gargallo, 2008). 
Toda renovación pedagógica comienza recurriendo a metodologías en las que se fomente la participación activa del alumnado (Rabadán \& Hernández, 2012) y para las que se requieren de formaciones específicas de carácter didáctico (Alba, 2005) que procuren a su vez el desarrollo de competencias interpersonales, la interacción social y la cooperación.

Por lo tanto, el objetivo del estudio es conocer la formación metodológica que ha recibido el profesorado universitario de la Región de Murcia, para verificar si existe relación entre la formación recibida durante el grado y posgrado, la formación continua de carácter didáctico y un mayor uso de dichos métodos de enseñanza.

\section{METODOLOGÍA}

\subsection{DISEÑOY PARTICIPANTES}

El diseño seguido es el de encuesta transversal, ex post facto, prospectivo de grupo único (Montero \& León, 2007).

Teniendo en cuenta que la población total del profesorado universitario de la Región de Murcia asciende a un total de 3192 docentes (1974 docentes en la Universidad de Murcia, 631 docentes en la Universidad Politécnica de Cartagena y 587 docentes en la Universidad Católica San Antonio de Murcia), se contó con una muestra representativa de 502 docentes, (49.50\% de la Universidad de Murcia, el $41.71 \%$ de la UCAM y el $8.78 \%$ final de la Universidad Politécnica de Cartagena). La media de edad fue de 42.98 años (DT $=9,785)$, siendo un $54.5 \%$ de hombres y un $46.49 \%$ de mujeres, con una media de años de experiencia docente de 13.3 años $(D T=10,03)$.

\subsection{INSTRUMENTO}

El instrumento utilizado en este estudio se incluyó dentro de una encuesta más amplia diseñada $a d$ hoc. para conocer la incidencia de la formación del profesorado universitario en el ejercicio docente, especialmente en lo referido al uso de los métodos de enseñanza. Para la elaboración de este instrumento se incluyeron una serie de ítems siguiendo el Métodos Delphi, en el que participaron ocho expertos de diferentes ramas, y una prueba piloto para comprobar el comportamiento de la encuesta en la muestra. En ella participaron 42 docentes de diferentes áreas, como: idiomas, educación, informática, ADE, nutrición, derecho, entre otros (Jiménez, Tornel \& González 2015). El resultado fue una encuesta formada por 6 ítems con escala de respuesta tipo Likert de cinco anclajes (Anexo I) que incluyó los métodos seleccionados por De Miguel (2006): trabajo cooperativo, lección magistral, trabajo por proyectos, estudio de casos, aprendizaje basado en problemas (ABP), resolución de ejercicios y problemas y el contrato de aprendizaje.

\subsection{ANÁLISIS}

En primer lugar, se calcularon estadísticos descriptivos (frecuencias, medias y desviaciones típicas), como parte inicial de la investigación.

En segundo lugar, se llevaron a cabo cálculos relativos a estadística inferencial. Concretamente, se realizaron correlaciones de Pearson para poner a prueba y conocer 
la relación entre variables cuantitativas y semi-cuantitativas para la formación recibida en el máster y doctorado y uso de métodos. Las correlaciones se consideran de pequeño tamaño cuando $r=.10$, moderadas cuando $r=.30$ y elevadas cuando $r=.50$ o superior (Cohen, 1988).

También se calcularon análisis de varianza multivariados (MANOVAs) para evaluar las diferencias en el centroide (vector) de medias de las múltiples variables dependientes en las diferentes categorías de las variables independientes. Éste fue el proceso en la puesta a prueba de la relación entre métodos y formación inicial del profesorado y la formación didáctica en el uso de métodos.

En este análisis, se escogió el criterio de Pillais, de entre los diversos criterios multivariados para evaluar los efectos, dado su carácter robusto frente al no cumplimiento de los supuestos (Tabachnick \& Fidell, 2007).

Además, se realizan pruebas post-hoc o ANOVAs de continuación en los casos en que la prueba $\mathrm{F}$ del MANOVA mostraba que el centroide (vector) de medias de las variables dependientes era diferente en los grupos formados por las categorías de las variables independientes. De este modo, se determina qué grupos muestran diferencias significativas en sus medias.

Por último, se incluyó el cálculo y evaluación de tamaños del efecto (eta-cuadrado parciales) basándose en las guías de Cohen (1992) para interpretar la magnitud de dichos efectos: .02 como efecto bajo, .13 como efecto moderado y .26 como efecto elevado.

Todos los análisis se realizaron con SPSS 19.0.

\subsection{RESULTADOS}

La primera de las cuestiones sobre formación y métodos fue para comprobar que un $76 \%$ de la muestra afirma haber recibido formación específica en metodologías, y que el (87.8\%) estaría dispuesto a seguir formándose en métodos de enseñanza.

En la Figura 1 se muestra la formación didáctica del profesorado en los aspectos relacionados con métodos de enseñanza, con una media de $2.91(D T=1.37)$ en innovación, buenas prácticas e intercambios docentes, un $2.86(D T=1.35)$ utilización de nuevas tecnologías y recursos para la enseñanza, $2.80(D T=1.39)$ formación relacionada con los cambios asociados al nuevo contexto universitario, $2.74(D T=1.30)$ para rol del profesorado para el desempeño profesional, $2.66(D T=1.28)$ en la realización de seminarios y talleres sobre metodologías de enseñanza y $2.57(D T=1.30)$ en los sistemas de evaluación. 
Figura 1. Formación didáctica del profesorado en aspectos relacionados con métodos de enseñanza

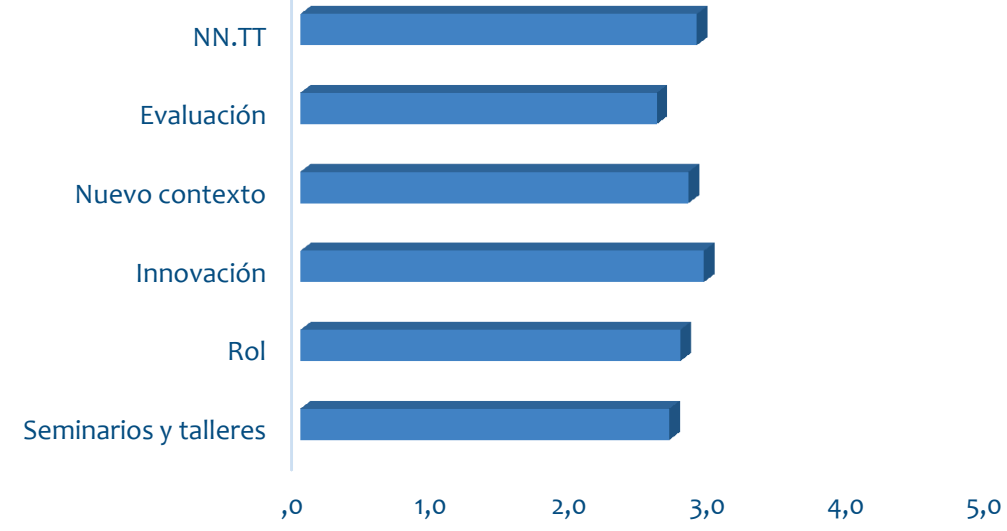

Nota: NN.TT = utilización de nuevas tecnologías y recursos para la enseñanza; Evaluación $=$ sistemas de evaluación; Nuevo contexto= formación relacionada con los cambios asociados al nuevo contexto universitario; Innovación = innovación, buenas prácticas e intercambios docentes; Rol= rol del profesorado para el desempeño profesional; Seminarios y talleres= en la realización de seminarios y talleres sobre metodologías de enseñanza.

Para conocer los métodos utilizados por el profesorado en función de la formación didáctica, se realizaron tres MANOVAs con las variables que medían si obtuvieron formación en metodología, la formación de grado y durante el máster y doctorado, una correlación con el tipo de formación didáctica en aspectos relacionados con estos métodos.

El primero de los MANOVAs puesto a prueba, que relacionaba al profesorado que había adquirido formación didáctica con el uso de éstos, resultó estadísticamente significativo en la combinación lineal de uso de métodos de enseñanza $\left(\mathrm{F}_{(7,491)}=4.213, p<.001, \eta^{2}=.06\right)$.

Los ANOVAs de continuación mostraron que, concretamente, existían diferencias estadísticamente significativas en el caso del trabajo cooperativo, trabajo por proyectos, estudio de casos y ABP (Tabla 1), con medias superiores en el caso de haber recibido formación, en todos los casos (Figura 2).

Tabla 1. ANOVAs de continuación para el MANOVA que relaciona la formación didáctica con dimensiones de los métodos como variables dependientes

\begin{tabular}{|l|l|c|c|c|c|c|}
\hline Fuente de variación & Variables dependientes & $\mathbf{g l}_{\mathbf{n u m}}$ & $\mathbf{g l}_{\mathbf{d e n}}$ & $\boldsymbol{F}$ & $\mathbf{p}$ & $\eta^{2}$ \\
\hline Formación didáctica & Trabajo cooperativo & 1 & 497 & 18.836 & .001 & .04 \\
\hline & Lección Magistral & 1 & 497 & .900 & .343 & .002 \\
\hline & Trabajo por proyectos & 1 & 497 & 8.491 & .004 & .02 \\
\hline & Estudio de casos & 1 & 497 & 10.523 & .001 & .02 \\
\hline & ABP & 1 & 497 & 7.416 & .007 & .015 \\
\hline & Resolución de problemas y ejercicios & 1 & 497 & 1.767 & .184 & .004 \\
\hline & Contrato de aprendizaje & 1 & 497 & 3.036 & .082 & .006 \\
\hline
\end{tabular}


Figura 2. Medias de sujetos formados en métodos de enseñanza frente a los que no en cada uno de los métodos

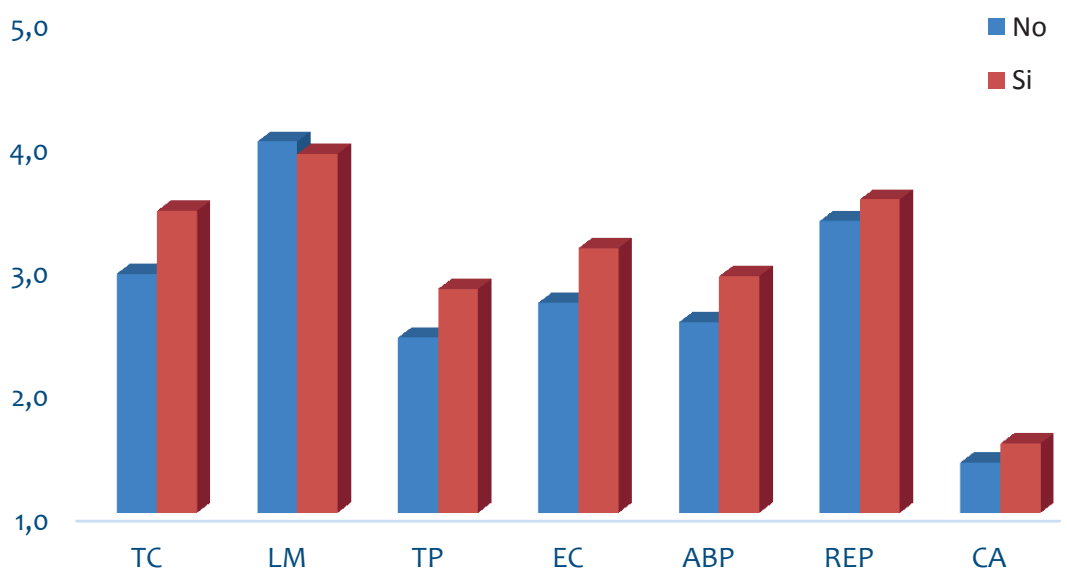

Nota: $\mathrm{TC}=$ Trabajo cooperativo; $\mathrm{LM}=$ lección magistral; $\mathrm{TP}=$ trabajo por proyectos; $\mathrm{EC}=$ estudio de casos; $\mathrm{ABP}=$ aprendizaje basado en problemas; $\mathrm{REP}=$ resolución de ejercicios y problemas; $\mathrm{CA}=$ contrato de aprendizaje .

Utilizando esta misma variable, se creó una nueva para resumir el tipo de formación didáctica. Al poner a prueba su relación con el uso general de los métodos se obtuvo una correlación estadísticamente significativa y positiva $(r=.326, p<.01)$.

$\mathrm{Al}$ pormenorizar el estudio y poner a prueba la relación entre el tipo de formación didáctica y el uso concreto de cada uno de los métodos propuestos (Tabla 2), se observó que en el caso de los tres primeros tipos de formación didáctica, es decir, realización de seminarios y talleres sobre metodologías de enseñanza, rol del profesorado para el desempeño profesional e innovación, buenas prácticas e intercambios docentes, obtuvieron correlaciones estadísticamente significativas y positivas con el trabajo cooperativo, trabajo por proyectos, estudio de casos, ABP y contrato de aprendizaje y negativas con la lección magistral.

La formación relacionada con los cambios asociados al nuevo contexto universitario y utilización de nuevas tecnologías y recursos para la enseñanza, estableció unas correlaciones estadísticamente significativas y positivas con el trabajo cooperativo, trabajo por proyectos, estudio de casos, ABP, resolución de problemas y ejercicios y contrato de aprendizaje.

Finalmente, con la formación los sistemas de evaluación se hallaron correlaciones estadísticamente significativas y positivas con el trabajo cooperativo, trabajo por proyectos, estudio de casos, ABP, resolución de ejercicios y problemas y contrato de aprendizaje y negativas con la lección magistral. 
Tabla 2. Correlaciones entre el tipo de formación didáctica y uso de métodos de enseñanza

\begin{tabular}{|c|c|c|c|c|c|c|c|}
\hline & TC & LM & $\mathbf{T P}$ & EC & $\mathbf{A B P}$ & REP & CA \\
\hline Seminarios y talleres & $.241 * *$ & $-.123 * *$ & $.206 * *$ & $.163 * *$ & $.173 * *$ & .064 & $.152 * *$ \\
\hline Rol & $.291 * *$ & $-.142 * *$ & $.195 * *$ & $.185 * *$ & $.195^{* *}$ & .071 & $.183^{* *}$ \\
\hline Innovación & $.324 * *$ & $-.129 * *$ & $.225 * *$ & $.179 * *$ & $.198 * *$ & .081 & $.177 * *$ \\
\hline Nuevo contexto & $.186^{* * *}$ & -.003 & $.153 * *$ & $.172 * *$ & $.175^{* *}$ & $.113 *$ & $.157 * *$ \\
\hline Evaluación & $.218 * *$ & $-.121 * *$ & $.163^{* *}$ & $.213 * *$ & $.249 * *$ & $.122 * *$ & $.212 * *$ \\
\hline NN.TT & $.240 * *$ & -.014 & $.184 * *$ & $.183 * *$ & $.150 * *$ & $.098^{*}$ & $.133 * *$ \\
\hline
\end{tabular}

Nota: $* \mathrm{p}<.05 ; * * \mathrm{p}<.01 . \mathrm{TC}=$ trabajo cooperativo $\mathrm{LM}=$ lección magistral; $\mathrm{TP}=$ trabajo por proyectos; $\mathrm{EC}=$ estudio de casos; $\mathrm{ABP}=$ aprendizaje basado en problemas; $\mathrm{REP}=$ resolución de ejercicios y problemas; $\mathrm{CA}=$ contrato de aprendizaje; Seminarios y talleres= en la realización de seminarios y talleres sobre metodologías de enseñanza; Rol= rol del profesorado para el desempeño profesional; Innovación = innovación, buenas prácticas e intercambios docentes; Nuevo contexto = formación relacionada con los cambios asociados al nuevo contexto universitario; Evaluación = sistemas de evaluación; NN.TT = utilización de nuevas tecnologías y recursos para la enseñanza.

El segundo de los MANOVAs que se calculó, puso a prueba la relación entre la formación recibida durante el grado (diferenciando entre diplomado, graduado, licenciado e ingeniero superior) y los métodos que utilizaba, para el que resultó estadísticamente significativo en la combinación lineal de uso de métodos de enseñanza $\left(\mathrm{F}_{(7,491)}=678.120\right.$, $p<.001, \eta^{2}=.907$; Figura 3).

En la Tabla 3 se muestran los ANOVAs de continuación, en la que se pueden apreciar las diferencias estadísticamente significativas en el trabajo cooperativo y en la resolución de ejercicios y problemas.

Tabla 3. ANOVAs de continuación para el MANOVA que relaciona la formación recibida durante el grado con los métodos de enseñanza

\begin{tabular}{|l|l|c|c|c|c|c|}
\hline Fuente de variación & Variables dependientes & $\mathrm{gl}_{\mathrm{num}}$ & $\mathrm{gl}_{\mathrm{den}}$ & $F$ & $\mathrm{p}$ & $\eta$ \\
\hline Formación grado & Trabajo cooperativo & 3 & 495 & 3.270 & .021 & .02 \\
\hline & Lección Magistral & 3 & 495 & 1.308 & .271 & .008 \\
\hline & Trabajo por proyectos & 3 & 495 & 2.398 & .067 & .014 \\
\hline & Estudio de casos & 3 & 495 & 2.448 & .063 & .015 \\
\hline & ABP & 3 & 495 & 1.514 & .210 & .009 \\
\hline & Resolución de problemas yejercicios & 3 & 495 & 14.244 & .001 & .079 \\
\hline & Contrato de aprendizaje & 3 & 495 & 1.018 & .384 & .006 \\
\hline
\end{tabular}


Las pruebas a post hoc muestran que, pese a que en el caso de trabajo cooperativo se hallaron diferencias estadísticamente significativas, cuando se hace el estudio por pares este efecto desaparece ( $p>$.05). Por tanto, no existen diferencias estadísticamente significativas en función de la formación inicial.

En el caso de resolución de problemas y ejercicios, las pruebas a posteriori muestran que existen diferencias estadísticamente significativas entre los profesores cuya formación en el grado ha sido una ingeniería superior y el resto $(\mathrm{p}<.05)$, con un uso mayor en el caso de los ingenieros, en todos los casos (Figura 3).

Figura 3. Medias de métodos de enseñanza en función de la formación de grado

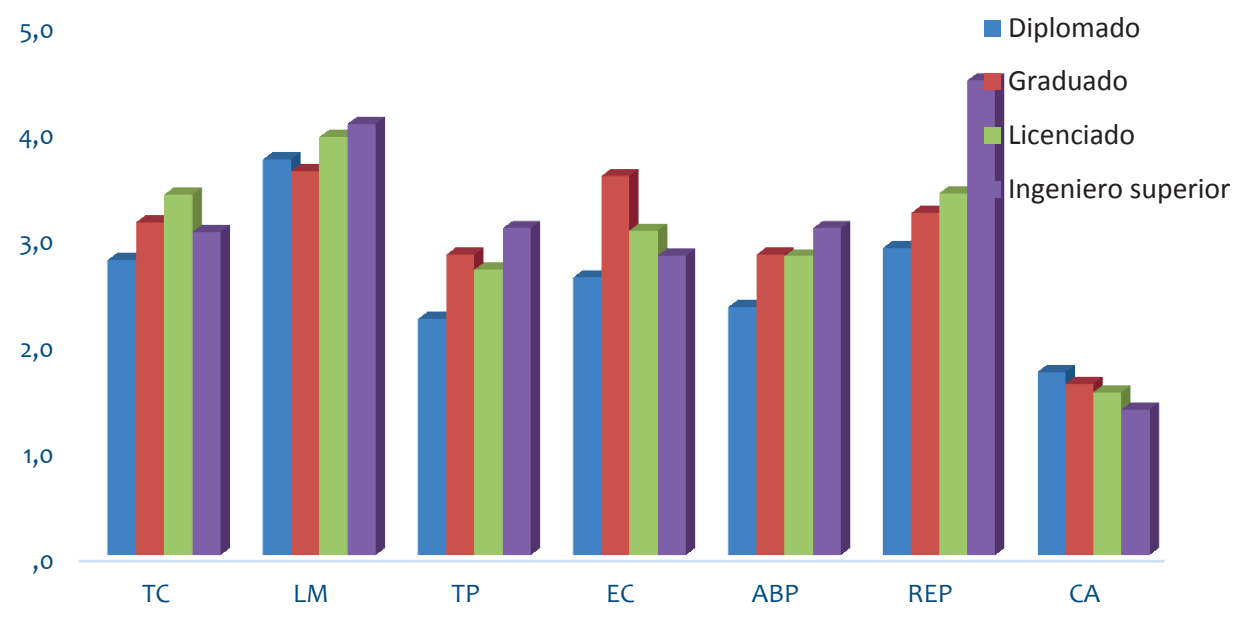

Nota: $\mathrm{TC}=$ trabajo cooperativo; $\mathrm{LM}=$ lección magistral; $\mathrm{TP}=$ trabajo por proyectos; $\mathrm{EC}=$ estudio de casos; $\mathrm{ABP}=$ aprendizaje basado en problemas; $\mathrm{REP}=$ resolución de ejercicios y problemas; $\mathrm{CA}=$ contrato de aprendizaje.

Para completar el estudio de estas variables, se puso a prueba la relación entre la formación durante el postgrado diferenciando entre máster y doctorado y el uso de métodos de enseñanza. Como se puede observar en la Tabla 4, la formación de máster y doctorado muestra una relación estadísticamente significativa y positiva con la utilización de la lección magistral, el ABP y la resolución de ejercicios y problemas. 
Tabla 4. Correlaciones entre las dimensiones uso y la formación de máster y doctorado.

\begin{tabular}{|l|c|}
\hline & Formación de máster y doctorado \\
\hline Trabajo Cooperativo & .067 \\
\hline Lección magistral & $.124 * *$ \\
\hline Trabajo por proyectos & .087 \\
\hline Estudio de casos & .050 \\
\hline Aprendizaje basado en problemas & $.134 * *$ \\
\hline Resolución de ejercicios y problemas & $.109 *$ \\
\hline Contrato de aprendizaje & -.028 \\
\hline
\end{tabular}

Nota: $* p<.05 ; * * p<.01$.

\section{DISCUSIÓN Y CONCLUSIONES}

Siguiendo la primera parte del objetivo de la investigación, se puede observar que tres de cada cuatro docentes afirman haber recibido formación metodológica. Estos porcentajes tan altos en la formación docente en métodos de enseñanza se remontan a los inicios del proceso de convergencia. Valcárcel (2004) realizó un estudio, en el que las universidades participantes respondieron que ya habían iniciado planes de formación del profesorado en el EEES. En el estudio llevado a cabo por Alba (2005), el 56\% de las universidades participantes pusieron en marcha acciones de aplicación de la metodología ECTS. Otras acciones formativas han sido los cursos sobre cuestiones metodológicas y estrategias docentes en un $25 \%$ de las universidades integrantes en el estudio. En esta modalidad, el Ministerio de Educación y Ciencia (2006) obtuvo un porcentaje mayor de formación en aspectos metodológicos y de naturaleza pedagógica para mejorar la calidad del aprendizaje, concretamente el $75 \%$.

De la muestra a la que se le realizó la encuesta, solo uno de cada cuatro docentes nunca ha recibido formación metodológica, la misma puntuación que Masjuan y Troiano (2009) obtuvieron del profesorado que se resistían a utilizar estos métodos por tener una valoración negativa, en palabras de sus autores, infundada por las mayores demandas de trabajo.

Continuando con la descripción de la formación recibida por el profesorado, destaca la formación didáctica como variable relacionada con el conocimiento y uso de metodologías de enseñanza.

Delgado (2013) extrajo que en la universidad de Burgos se forman a los docentes en metodologías adaptadas al EEES a través de la formación permanente. Sin embargo, también reconoce formaciones relacionadas con la renovación de metodologías docentes. No obstante, en Alba \& Carballo (2005) los docentes demandan formación de manera genérica sobre el EEES y más específicamente sobre las nuevas metodologías didácticas con el objetivo de favorecer su cambio. La formación en estos métodos también lo observó De Pablos (2007) en las necesidades formativas del profesorado de la Universidad de Sevilla. Sánchez (2006) por su parte, comprobó que las temáticas sobre las que más se ha 
formado el profesorado en la universidad pública tienen que ver con métodos pedagógicos para la docencia y con asuntos vinculados al EEES.

Conociendo las medias de formación didáctica y que tres de cada cuatro docentes han recibido formación en estos métodos, no resulta llamativo que un porcentaje aún mayor estaría dispuesto a seguir formándose. Síntoma de que su formación no fue la suficiente o que, una mayor asistencia de los docentes a cursos de formación se traduce en más asistencia a otros nuevos al sentir una mayor necesidad de seguir formándose (Zabalza, 2003-2004).

Contrariamente a lo expuesto y a los resultados que se han obtenido, en otros estudios como el de Bosco \& Rodríguez (2008), se advierte que el profesorado apenas tiene formación suficiente para desarrollar propuestas metodológicas alternativas a la clase magistral. Luque \& Navarro (2011) también observaron que se precisa de una mayor formación de los docentes universitarios en el ámbito de las técnicas y metodologías afines al EEES. Quizás, debido a que una gran parte de los docentes que se hayan formado en estos métodos quieran seguir formándose, como dicen estos autores, por haber obtenido una formación escasa para poder aplicar estos métodos en la práctica.

Como ha quedado descrito, existe un gran bagaje de investigaciones que han puesto de manifiesto cómo con el proceso de convergencia aumentó considerablemente la formación sobre métodos de enseñanza. Lo que también explica que el profesorado después de esta formación se sienta más preparado para utilizar dichos métodos que con su formación de grado.

A nivel internacional, existen investigaciones que avalan que una mayor formación de los docentes universitarios deriva en un mayor empleo de métodos de enseñanza, aunque explicado en dos fases. Una mayor formación deriva en un mayor enfoque centrado en el alumnado (Postareff, Lindblom-Ylänne \& Nevgi 2008; Gibbs \& Coffey 2004) y que este enfoque, repercute en la mayor utilización de diferentes métodos de enseñanza (Light, Calkins, Luna \& Drane 2009; Drew 2004; Trigwell \& Prosser 2004; Hativa 2000).

Continuando con el objetivo de investigación, se cuestionó si una mayor formación didáctica, durante grado y el máster y el doctorado, desembocaría también en un mayor uso de métodos de enseñanza en comparación al profesorado que ha obtenido poca o ninguna formación.

Los resultados muestran relaciones significativas para todos los aspectos de la formación didáctica. Ahora bien, el profesorado que sí ha obtenido formación didáctica utilizaba más el trabajo cooperativo, trabajo por proyectos, estudio de casos y ABP (la mayoría de métodos activos). Un ejemplo de estudio sobre formación del profesorado en métodos de enseñanzas con resultados semejantes fue el programa ERAGIN. Un programa de formación en co-mentoría sobre métodos como el estudio de casos, ABP y trabajo por proyectos (Fernández, Guisasola, Garmendia, Alkorta \& Madinabeitia 2013). Existe diversas experiencias en nuestro país sobre cursos y talleres de esta temática (Velázquez, Alducin, Marín \& Cabero 2012; Gallardo, 2009-2010; Valcárcel 2003).

Con respecto al tipo de formación didáctica, a nivel general obtuvo una relación significativa y positiva con el uso global de los métodos de enseñanza. En cada uno de los tipos de formación el resultado fue bastante parecido, exceptuando la resolución de ejercicios y problemas que no tenía este tipo de relaciones y la lección magistral que en 4 de los 6 tipos obtuvo una relación significativa y negativa (este método es el menos activo y más común de los propuestos). Esto evidencia la relación existente entre la formación didáctica en los distintos tipos propuestos, con la aplicación variada de métodos de enseñanza por parte de los docentes consultados y menor con el uso de la lección magistral. 
Es decir, este tipo de formación favorece que el profesorado emplee métodos de enseñanza menos habituales en su docencia y que requieren una mayor implicación activa y autónoma del estudiante en el proceso de enseñanza-aprendizaje.

Entre los estudios realizados en el contexto español acerca de la formación en métodos de enseñanza y cuyos resultados concuerdan con los expuestos, se encuentra el Ministerio de Educación y Ciencia (2005), en el que un mayor empleo de métodos de enseñanza tiene lugar cuando se tienen procesos de formación didáctica.

La formación de grado (diplomado, graduado, licenciado e ingeniero superior), resultó significativa con el uso general de métodos, siendo el tamaño del efecto hallado entre ambas variables grande. Los análisis posteriores, revelan una relación entre el uso de la resolución de problemas y ejercicios por parte de los ingenieros superiores frente al resto de áreas. Estos resultados van en consonancia con los hallados en otras investigaciones. Así, De Miguel (2005) ya mencionó que las ingenierías tienen tradición en la realización de proyectos, ensayos y simulaciones para trabajar habilidades intelectuales conectadas con la realización de tareas reales. También el Ministerio de Educación y Ciencia (2006) confirmó que las Ingenierías recurrían a metodologías activas y motivadoras para acercar a la realidad profesional de la etapa y adquirir capacidades científico-técnicas de la profesión.

Los resultados exponen que, a mayor formación durante el postgrado, diferenciando entre máster y doctorado, hay un mayor uso de la lección magistral, ABP y resolución de problemas y ejercicios. Al ser la lección magistral uno de los más aplicados por el profesorado y menos activos para el alumnado, no se puede decir de una manera tan clara como la formación didáctica, que la formación durante el postgrado sea un indicador de una mayor utilización de metodologías de enseñanza más innovadoras.

Caballero (2013) coincide con esta investigación en que la formación recibida durante el máster y el doctorado para ser docente, influye menos en el desarrollo de su actividad profesional que la formación específica en cuestiones didácticas. Así mismo, también coincide en que los docentes que se encontraban más capacitados para aplicar diferentes métodos de enseñanza en sus clases con la formación que tienen actualmente que con la que obtuvieron al terminar sus estudios universitarios.

González et al. (2009) atribuyeron a la formación de grado y a la específica del profesorado universitario el reto del cambio metodológico y, como se puede observar, en la formación didáctica sí se cumple dicha afirmación. En la formación recibida en el grado solo en algunos aspectos.

Estos resultados apuntan a que, además de la experiencia, para la transformación de las clases, es necesaria una mayor formación en el conocimiento y dominio de otras formas de enseñanza (Zabalza, 2003-2004).

Ha quedado retratado cómo la formación que recibe el profesorado, especialmente la didáctica, influye en su docencia, y más concretamente en el empleo de diferentes métodos de enseñanza. Esta formación se vio acrecentada por la inclusión del sistema universitario en el EEES y, por la tasa de participación, queda evidenciado que el profesorado se implica en procesos formativos para mejorar su docencia. De esta manera, la formación del profesorado sigue siendo un factor fundamental en los procesos de cambio educativos y renovación pedagógica. No obstante, teniendo en cuenta las limitaciones relacionadas con la muestra, queda patente la necesidad de investigar en mayor profundidad este tema para detectar las posibles causas que llevan en última instancia al uso de métodos de enseñanza por parte de los docentes, apuntando así las áreas de intervención que favorezcan el cambio. 


\section{ENCUESTA}

\begin{tabular}{|c|c|c|c|c|c|c|}
\hline $\begin{array}{l}\text { Formación Universitaria recibida: } \\
\text { Diplomado } \\
\text { Licenciado } \\
\text { Ingeniero Técnico } \\
\text { Ingeniero Superior } \\
\text { Graduado }\end{array}$ & $\begin{array}{l}\text { Formación } \\
\text { CAP } \\
\text { Máster }\end{array}$ & oste & & & & \\
\hline \multicolumn{5}{|c|}{$\begin{array}{l}\text { 1. ¿Ha recibido en alguna ocasión formación específica sobre métodos didácticos } \\
\text { activos? }\end{array}$} & $\mathrm{Si}$ & No \\
\hline \multicolumn{7}{|c|}{$\begin{array}{l}\text { 2. ¿Ha recibido formación específica sobre alguno de estos aspectos? (valore del } 1 \text { al } 5 \text {, teniendo } \\
\text { en cuenta que el valor } 1 \text { corresponde a poca y el } 5 \text { a mucha) }\end{array}$} \\
\hline A & $\begin{array}{l}\text { Implementación y } \\
\text { aplicación de métodos } \\
\text { activos }\end{array}$ & 1 & 2 & 3 & 4 & 5 \\
\hline B & $\begin{array}{l}\text { Rol del profesorado en } \\
\text { métodos activos }\end{array}$ & 1 & 2 & 3 & 4 & 5 \\
\hline $\mathrm{C}$ & $\begin{array}{l}\text { Innovación y buenas } \\
\text { prácticas con métodos } \\
\text { activos }\end{array}$ & 1 & 2 & 3 & 4 & 5 \\
\hline $\mathrm{D}$ & $\begin{array}{l}\text { Adaptación a la } \\
\text { dinámica del EEES }\end{array}$ & 1 & 2 & 3 & 4 & 5 \\
\hline $\mathrm{E}$ & $\begin{array}{l}\text { Evaluación de } \\
\text { métodos activos }\end{array}$ & 1 & 2 & 3 & 4 & 5 \\
\hline $\mathrm{F}$ & $\begin{array}{l}\text { TIC aplicadas a } \\
\text { métodos activos }\end{array}$ & 1 & 2 & 3 & 4 & 5 \\
\hline \multicolumn{7}{|c|}{$\begin{array}{l}\text { 3. Valore con qué frecuencia recurre a estos métodos activos (valore del } 1 \text { al } 5 \text {, teniendo en cuenta } \\
\text { que el valor } 1 \text { corresponde a nunca y el } 5 \text { a siempre) }\end{array}$} \\
\hline A & Trabajo cooperativo & 1 & 2 & 3 & 4 & 5 \\
\hline B & Lección magistral & 1 & 2 & 3 & 4 & 5 \\
\hline $\mathrm{C}$ & Trabajo por proyectos & 1 & 2 & 3 & 4 & 5 \\
\hline $\mathrm{D}$ & Estudio de casos & 1 & 2 & 3 & 4 & 5 \\
\hline $\mathrm{E}$ & $\begin{array}{l}\text { Aprendizaje basado en } \\
\text { problemas (ABP) }\end{array}$ & 1 & 2 & 3 & 4 & 5 \\
\hline $\mathrm{F}$ & $\begin{array}{l}\text { Resolución de } \\
\text { ejercicios y problemas }\end{array}$ & 1 & 2 & 3 & 4 & 5 \\
\hline G & $\begin{array}{l}\text { Contrato de } \\
\text { aprendizaje }\end{array}$ & 1 & 2 & 3 & 4 & 5 \\
\hline $\mathrm{H}$ & \multicolumn{6}{|c|}{$\begin{array}{l}\text { ¿Recurre a otro/s método/s activos que no hayamos } \\
\text { incluido? ¿Cuál/es? }\end{array}$} \\
\hline 4. ¿Estaría dispuesto a formarse o & rmándose en métodos & & & & $\mathrm{Si}$ & No \\
\hline
\end{tabular}




\section{REFERENCIAS BIBLIOGRÁFICAS}

Alba, C. (2005). La universidad española en el EEES, el profesorado universitario y las TIC en el proceso de Convergencia Europea. Revista de Educación, (337), 7-11.

Alba, C., \& Carballo, R. (2005). Viabilidad de las propuestas metodológicas para la aplicación del crédito europeo por parte del profesorado de las universidades españolas, vinculadas a la utilización de las TIC en la docencia y la investigación. Revista de Educación, 337, 71-97.

Arribas, J. M., Carabias, D. \& Monreal, I. (2010). La docencia universitaria en la formación inicial del profesorado. El caso de la escuela de magisterio de Segovia. Revista electrónica Interuniversitaria de Formación del Profesorado, 13(3), 27-35.

Bosco, A. \& Rodríguez, D. (2008). Docencia Virtual y Aprendizaje Autónomo. Algunas contribuciones al Espacio Europeo de Educación Superior. RIED: revista iberoamericana de educación a distancia, 11(1), 157-182.

Caballero, K. (2013). La formación del profesorado universitario y su influencia en el desarrollo de la actividad profesional. REDU. Revista de Docencia Universitaria, 11(2), 391-412.

Cohen, J. (1992). A power primer. Quantitative Methods in Psychology, 112(1), 155-159.

Cohen, J. (1988). Statistical power analysis for the behavioral sciences (2nd Ed.). New Jersey: Lawrence Erlbaum.

De la Calle, M. J. (2004). El Reto de ser profesor en el contexto de la convergencia europea. La formación pedagógica como necesidad. Revista Interuniversitaria de Formación del Profesorado, 18(3), 251-258. Recuperado de http://aufop.com/aufop/uploaded_files/articulos/1212408779.pdf

De Miguel, M. (2006). Modalidades de enseñanza centradas en el desarrollo de competencias. Orientaciones para promover el cambio metodológico en el marco del EEES. Asturias: Universidad de Oviedo.

De Miguel, M. (2005). Cambio de paradigma metodológico en la Educación Superior. Exigencias que conlleva. Cuadernos de integración europea, 2, 16-27.

De Pablos, J. (2007). El cambio metodológico en el espacio europeo de educación superior y el papel de las tecnologías de la información y la comunicación. Revista Iberoamericana de Educación a Distancia, 10(2). Recuperado de http://e-spacio.uned.es/revistasuned/index.php/ried/article/ view/992

Delgado, V. (2013). La formación del profesorado universitario. Análisis de los programas formativos de la Universidad de Burgos (2000-2011) (Universidad de Brugos). Burgos, España.

Drew, L. (2004). The experience of teaching creative practices: conceptions and approaches to teaching in the community of practice dimension. Recuperado de http://ualresearchonline.arts. ac.uk/644/1/cltad_ped_res_pap_linda_drew.pdf

Escudero, J. M. \& Portela, A. (2015). La formación continuada del profesorado: políticas, programas, aprendizajes docentes e impacto en la enseñanza y los aprendizajes del alumnado. En M. A. Flores y F. Ilídio (eds.), Formaçao e Trabalho docente (pp. 25-37). Santo Tirso: De Facto Editores.

Fernández, I., Guisasola, G., Garmendia, M., Alkorta, I. \& Madinabeitia, A. (2013). ¿Puede la formación tener efectos globales en la universidad? Desarrollo docente, metodologías acticas y curriculum híbrido. Infancia y aprendizaje, 36(3), 387-400.

Gallardo, D. (2009-2010). Plan de formación del profesorado Universidad de Extremadura (20092010). Badajoz: Servicio de Orientación y Formación Docente Universidad de Extremadura.

Gargallo, B. (2008). Estilos de docencia y evaluación de los profesores universitarios y su influencia sobre los modos de aprender de sus estudiantes. Revista Española de Pedagogía, 425-445.

Gibbs, G. \& Coffey, M. (2004). The impact of training of university teachers on their teaching skills, their approach to teaching and the approach to learning of their students. Active learning in higher education, 5(1), 87-100. Recuperado de http://alh.sagepub.com/content/5/1/87.short

González, F. E., Macías, E., Rodríguez, M. \& Aguilera, J. L. (2009). Prospectiva y evaluación del 
ejercicio docente de los profesores universitarios como exponente de buena calidad. Revista de universidad y Sociedad del conocimiento, 6(2), 38-48.

González, M. \& Raposo, M. (2008). Necesidades formativas del profesorado universitario en el contexto de la convergencia europea. Revista de Investigación, 26(2), 285-306.

Gore, J., Griffiths, T. \& Ladwing, J. G (2004). Towards better teaching: productive pedagogy as a framework for teacher education. Teaching and Teacher Education, 20, 375-387

Hativa, N. (2000). Teaching for Effective Learning in Higher Education. Dordrecht: Kluwer Academic Publishers.

Jarauta, B. \& Medina, J. L. (2012). Fuentes y procesos de aprendizaje docente en el contexto universitario. Revista Española de Pedagogía, 337-353.

Jiménez, D., Tornel, M. \& González, J. J. (2015). Optimización de un cuestionario mediante un método Delphi y una prueba piloto. Opción, (4), 717-638.

Kivunja, C. (2014) Innovative Pedagogies in Higher Education to Become Effective Teachers of 21st Century Skills: Unpacking the Learning and Innovations Skills Domain of the New Learning Paradigm. International Journal of Higher Education, 3(4), 37-48.

Ladwig, J. (2005) Monitoring the Quality of Pedagogy. Leading \& Managing, 11(2), 70-83.

Light, G., Calkins, S., Luna, M. \& Drane, D. (2009). Assessing the Impact of a Year-Long Faculty Development Program on Faculty Approaches to Teaching. International Journal of Teaching and Learning in Higher Education, 20(2), 168-181.

López, E. (2016) La formación docente del profesorado universitario: sentido, contenido y modalidades. Bordón, 68(4), 89-102.

Luque, A. M. \& Navarro, E. (2011). El aprendizaje cooperativo y la enseñanza de la geografía en el marco del EEES. Didáctica Geográfica, (12), 49-72.

Madrid, J. M. (2005). La formación y la evaluación docente del profesorado universitario ante el espacio europeo de educación superior. Educatio Siglo XXI, 23, 49-68.

Masjuan, J. M. \& Troiano, H. (2009). Riesgos de fracaso en el proceso de implantación del espacio europeo de educación superior: La experiencia de una universidad catalana. Revista de la educación superior, 38(149), 73-94.

Michavila, F. (2005). No sin los profesores. Revista de Educación, (337), 37-49.

Ministerio de Educación y Ciencia (2006). Propuestas para la renovación de las metodologías educativas en la universidad. Recuperado de http://www.upcomillas.es/eees/Documentos/ PROPUESTA_RENOVACION.pdf

Ministerio de Educación y Ciencia (2005). El estado actual de las metodologías educativas en las universidades españolas. Recuperado de http://diposit.ub.edu/dspace/bitstream/2445/1110/1/ el $\% 20$ estado $\% 20$ actual $\% 20$ de $\% 201$ as $\% 20$ metodologies $\% 20$ educativas $\% 20$ en $\% 201$ as $\% 20$ universidades\%20uropeas.pdf

Monereo, C. (2013). La investigación en la formación del profesorado universitario: hacia una perspectiva integradora. Infancia y Aprendizaje, 36(3), 281-291.

Montero, I. \& León, O. G. (2007). A guide for naming research studies in Psychology. International Journal of Clinical and Health Psychology, 7, 847-862.

Postareff, L., Lindblom-Ylänne, S. \& Nevgi, A. (2008). A follow-up study of the effect of pedagogical training on teaching in higher education. Higher Education, 56(1), 29-43. Recuperado de http:// link.springer.com/article/10.1007/s10734-007-9087-z

Pozo, C., Bretones, B., Martos, M. J. \& Alonso, E. (2011). Evaluación de la actividad docente en el Espacio Europeo de Educación Superior: un estudio comparativo de indicadores de calidad en universidades europeas. Revista Española de Pedagogía, 145-163.

Rabadán, J. A. \& Hernández, E. (2012). Renovación pedagógica en la Sociedad del Conocimiento. Nuevos retos para el profesorado universitario. RED-DUSC, Revista de Educación a Distancia. Sección de Docencia Universitaria en la Sociedad del Conocimiento, (6). Recuperado de http:// www.um.es/ead/reddusc/6/rabadan.pdf 
Sánchez, F. (2006). Modelos de formación del profesorado y su valoración. Informe de resultados. Madrid: Universidad Autónoma de Madrid.

Tabachnick, G. G. \& Fidell, L. S. (2007). Using Multivariate Statistics (6th Ed.). Boston: Allyn and Bacon.

Trigwell, K. \& Prosser, M. (2004). Development and use of the approaches to teaching inventory. Educational Psychology Review, 16(4), 409-424.

Valcárcel, M. (2004). Diseño y validación de actividades de formación para profesores y gestores en el proceso de armonización europea en educación superior. (Estudio EA2004-0036). Córdoba: Ministerio de Educación y Ciencia. Recuperado de http://campus.usal.es/ ofeees/ESTUDIOS_ INFORMES_GRALES/informe_validacion_activ.pdf

Valcárcel, M. (2003). La preparación del profesorado universitario español para la convergencia europea en educación superior. (Proyecto EA2003-0040). Córdoba: Ministerio de Educación y Ciencia. Recuperado de http://campus.usal.es/ ofeees/ESTUDIOS_INFORMES_GRALES/ informe_final.pdf

Velázquez, A., Alducin, J., Marín, V. \& Cabero, J. C. (2012). Formación del profesorado para el Espacio Europeo de Educación Superior. Aula abierta, 40(2), 25-38. Recuperado de http:// dialnet.unirioja.es/servlet/articulo?codigo=3920896

Zabalza, M. A., Cid, A. \& Trillo, F. (2014). Formación docente del profesorado universitario. El difícil tránsito a los enfoques institucionales. Revista Española de Pedagogía, 39-54.

Zabalza, M. A. (2003-2004). Innovación en la enseñanza universitaria. Contextos educativos, (6-7), 113-136. 